\title{
Coherent Cooper-pair pumping by magnetic flux control
}

\author{
S. Gasparinetti \\ Low Temperature Laboratory, Aalto University, P.O. Box 15100, FI-00076 Aalto, Finland \\ I. Kamleitner \\ Institut für Theory der Kondensierten Materie, Karlsruher Institut für Technologie, 76128 Karlsruhe, Germany
}

\begin{abstract}
We introduce and discuss a scheme for Cooper-pair pumping. The scheme relies on the coherent transfer of a superposition of charge states across a superconducting island and is realized by adiabatic manipulation of magnetic fluxes. Differently from previous implementations, it does not require any modulation of electrostatic potentials. We find a peculiar dependence of the pumped charge on the superconducting phase bias across the pump and that an arbitrarily large amount of charge can be pumped in a single cycle when the phase bias is $\pi$. We explain these features and their relation to the adiabatic theorem.
\end{abstract}

\section{INTRODUCTION}

A Cooper-pair pump ${ }^{1}$ is a superconducting device that can be used to transport Cooper pairs by manipulating some of its parameters in a periodic fashion. Cooper-pair pumps have recently attracted considerable theoretical ${ }^{1-16}$ and experimental ${ }^{17}[22$ attention.

Part of this attention stems from the geometric properties of the parametric cycle used to perform pumping. These properties, in turn, leave a distinctive fingerprint in the pumped charge. The link between geometric phases and pumped charge has been established in the adiabatic limit, where an explicit relation connects the pumped charge to the Berry phase $e^{23 \mid 24}$, as first shown in Ref. 3 and experimentally demonstrated in Ref. 20. In addition, the breakdown of adiabatic behavior due to Landau-Zener transitions can be detected as a decrease in the pumped charge ${ }^{22}$. This offers the opportunity to develop Landau-Zener-Stückelberg interferometry ${ }^{25}$ based on geometric phases 13 . Finally, it has been proposed to exploit Cooper-pair pumps for the observation of nonabelian geometric phases $11 \mid 12$.

Another reason to study Cooper-pair pumps is that they are convenient solid-state implementations of a driven quantum two-level system. In the presence of a dissipative environment, the pumped charge is determined by the quasistationary state reached by the system and thus is a sensitive probe of decoherence effects. This explains why a Cooper-pair pump was chosen as a "case in point" in several theoretical works aimed at studying the role of dissipation in driven quantum systems $14|26| 28$.

Different types of Cooper-pair pumps have been proposed and realized $\frac{17 / 18 \mid 21}{}$. All these devices comprise the same building blocks, namely, superconducting islands connected to each other and to superconducting leads by Josephson junctions. They are intended to be operated in a regime where the charging energy of the islands is much larger than the Josephson energies of the couplings. Thus, at the heart of these implementations is the "classical" phenomenon of Coulomb blockade. Pumping relies on periodic modulation of electrostatic potentials, tuned by gate electrodes which act as pistons in pulling Cooper pairs onto and off the islands. The main contribution to pumping comes from incoherent tunneling of Cooper pairs 5 , with phase-coherent effects only providing small corrections.

In this Article, we undertake a different approach to Cooper-pair pumping, that we call "flux pumping" (FP). FP is based on the coherent transfer of a superposition of charge states across a superconducting island by adiabatic manipulation of magnetic fluxes. Contrary to previous proposals, FP does not involve the modulation of electrostatic potentials. The pumped charge resulting from FP is purely coherent. Its dependence on the phase bias across the pump reveals intriguing features. Among them, we find that for a particular choice of the system parameters, an arbitrarily large charge can be pumped per cycle. However, this is by no means inconsistent as at the same time the adiabatic criterion requires the pumping cycle to be correspondingly slow.

The device we consider for FP uses the same hardware as the Cooper pair sluice introduced in Ref. 4. Yet pumping is achieved in a completely different manner. First, the gate voltage is kept constant throughout the pumping cycle. Second, the opening times of the superconducting quantum interference devices (SQUIDs) used as valves have a large overlap. FP also differs from Cooper-pair shuttling $2 \sqrt{26}$, in at least two respects. First, while the "shuttle" is only coupled to one lead at a time (hence its name), in our case simultaneous coupling of the central island to both leads is required to achieve a nonvanishing pumped charge. Second, the operation of the shuttle is non-adiabatic and requires accurate control of the time dependence of the pulses. By contrast, FP is insensitive to the speed at which the cycle is performed, as long as the modulation is adiabatic. This is a consequence of the geometric nature of the pumped charge.

Features such as the large overlap between the flux pulses, the subordinate role played by Coulomb blockade, and the overall coherence of the pumping process, bring FP close together with pumping in open systems, 29 sometimes referred to as "quantum pumping". FP thus opens a new possibility to explore quantum pumping in superconducting systems. 
(a)

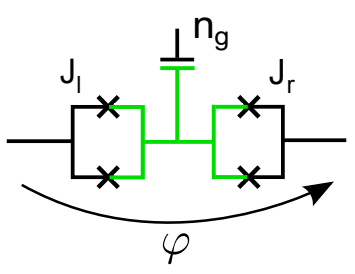

(b)

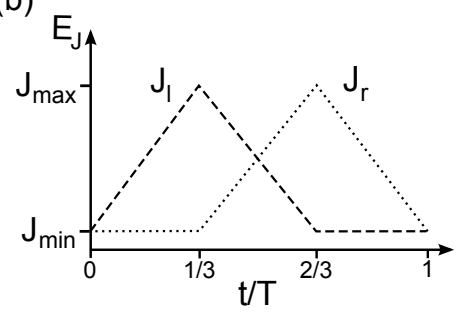

FIG. 1. (Color online) Pumping with magnetic fluxes. (a) Schematic circuit of a Cooper pair sluice. A superconducting island (green) is coupled to superconducting leads by two SQUIDs, acting as tunable Josephson junctions of energy $J_{l}$, $J_{r}$. A gate capacitively coupled to the island controls its polarization charge $n_{g}$. The superconducting phase of the two leads is held at a fixed difference $\varphi$. (b) Representative time modulation of $J_{l}, J_{r}$ leading to FP. The gate position is kept fixed throughout the modulation.

FP can also be connected to some adiabatic transfer schemes used in quantum information, in particular, the coherent transfer by adiabatic passage (CTAP) protoco ${ }^{32} 35$. In both schemes, the transfer relies on time-dependent manipulation of tunnelling rates rather than energy levels. However, only in FP is the device connected to leads, thereby allowing for the generation of a continuous pumped current. The presence of superconducting leads and their phase bias introduce novel features that have no equivalent in CTAP. A hallmark of CTAP is the so-called counter-intuitive pulse ordering: in order to transfer information from left to right, the right tunnel junction is operated first. In FP we do find a similar behavior when the phase bias is close to $\pi$; in general, however, current flows in the same direction as the pulse sequence.

The outline of this paper is as follows. In Sec. III we introduce the Cooper-pair sluice and set up the theoretical framework on which our calculations are based. In the core Sec. III, we describe flux pumping. In Sec. IV] we characterize the breakdown of adiabatic behavior by performing numerical simulations with a master equation approach. Finally, in Sec. V], we summarize our results and comment on the feasibility of our proposal.

\section{THE COOPER-PAIR SLUICE}

A schematic drawing of the Cooper-pair sluice is shown in Fig. 1 (a). It is a fully tunable Cooper-pair transistor, consisting of a small superconducting island connected to leads by two SQUIDs. The SQUIDs are controlled independently by adjusting the magnetic fluxes $\Phi_{l}, \Phi_{r}$ threading their loops, so that they can serve as Josephson junctions of tunable energy $J_{l}, J_{r}$. A gate electrode capacitively coupled to the island controls its polarization charge in units of Cooper pairs $n_{g}=C_{g} V_{g} / 2 e$, where $C_{g}$ is the cross-capacitance between gate and island and $V_{g}$ the gate voltage.

We assume that the superconducting electrodes to which the sluice is connected are held at a fixed phase difference $\varphi$. The simplest way to accomplish this is to embed the sluice in a superconducting loop. An other possibility is to shunt the sluice with a large Josephson junction of energy $J_{S} \gg J_{l}, J_{r}$. In this configuration, the Josephson junction can also serve as a current threshold detector. This technique was first applied to the readout of the "quantronium" circuit ${ }^{36}$ and then proposed for ${ }^{7}$ and successfully applied $\mathrm{tc}^{20}$ the sluice.

We use the sluice in the regime where the charging energy $E_{C}=4 e^{2} / 2 C_{\Sigma}\left(C_{\Sigma}\right.$ is the total island capacitance) is large compared to $J_{l}$ and $J_{r}$. We describe the dynamics in the basis of eigenstates of charge on the island, and restrict the Hilbert space to the states $|0\rangle$ and $|1\rangle$ with no and one excess Cooper pair on the island, respectively. In this two-level approximation, the Hamiltonian is given in matrix form by ${ }^{1}$

$$
\hat{H}=\left(\begin{array}{cc}
E_{C}\left(\frac{1}{2}+\delta n_{g}\right)^{2} & J_{+} \cos \frac{\varphi}{2}+i J_{-} \sin \frac{\varphi}{2} \\
J_{+} \cos \frac{\varphi}{2}-i J_{-} \sin \frac{\varphi}{2} & E_{C}\left(\frac{1}{2}-\delta n_{g}\right)^{2}
\end{array}\right)
$$

where $J_{ \pm}=\frac{1}{2}\left(J_{l} \pm J_{r}\right)$, and $\delta n_{g}=n_{g}-\frac{1}{2}$ the offset between the gate charge and the degeneracy point.

We now outline how to obtain the pumped charge in the adiabatic limit. We use the same notation as in Ref. 27, to which the reader is referred for a more detailed account. We also set $\hbar=1$ and $2 e=1$.

A pumping cycle is described by a closed loop in the space of a minimal set of parameters determining $\hat{H}$. Under the assumption that the parameters are changed slow enough, the system will approximately follow the instantaneous ground state of $\hat{H}$. This fact underlies the adiabatic theorem and is at the basis of a perturbation expansion. The latter is formally accomplished by introducing a local adiabatic parameter

$$
\alpha(t)=|\langle\dot{g}(t) \mid e(t)\rangle| / \Delta(t),
$$

where $|g(t)\rangle$ and $|e(t)\rangle$ are, respectively, the instantaneous ground and excited state of $\hat{H}$ (adiabatic states) and $\Delta(t)$ is the instantaneous energy gap at time $t$. The adiabatic limit is attained provided $\alpha(t) \ll 1$ at all times.

We will find use for the following quantities:

$$
\begin{gathered}
E_{12}=\frac{1}{2} \sqrt{J_{l}^{2}+J_{r}^{2}+2 J_{l} J_{r} \cos \varphi}, \\
\gamma=\arctan \left(\frac{J_{r}-J_{l}}{J_{r}+J_{l}} \tan \frac{\varphi}{2}\right), \\
\eta=\frac{\delta n_{g}}{\sqrt{\delta n_{g}^{2}+\left(\frac{E_{12}}{E_{C}}\right)^{2}}} .
\end{gathered}
$$

In terms of the fixed $\{|0\rangle,|1\rangle\}$ basis, the adiabatic states are explicitly given by:

$$
\begin{aligned}
& |g\rangle=\frac{1}{\sqrt{2}}\left(\sqrt{1-\eta}|0\rangle+e^{-i \gamma} \sqrt{1+\eta}|1\rangle\right), \\
& |e\rangle=\frac{1}{\sqrt{2}}\left(\sqrt{1+\eta}|0\rangle-e^{-i \gamma} \sqrt{1-\eta}|1\rangle\right) .
\end{aligned}
$$


In order to properly account for the pumped charge, it is essential to consider the corrections to the instantaneous ground state up to first order in $\alpha$. The resulting density matrix of the sluice, expressed in the adiabatic basis, is given by

$$
\begin{aligned}
& \rho_{g g}=1, \\
& \rho_{g e}=\frac{i \partial_{t} \eta-\left(1-\eta^{2}\right) \partial_{t} \gamma}{4 E_{12}} .
\end{aligned}
$$

After solving the dynamics, we can turn to the calculation of the pumped charge. We introduce current operators $\hat{I}_{k}$ for the $k$-th SQUID $(k=l, r)$. In the $\{|0\rangle,|1\rangle\}$ basis, one has:

$$
\begin{aligned}
\hat{I}_{l} & =\frac{J_{l}}{2 i}\left(\begin{array}{cc}
0 & -e^{-i \varphi / 2} \\
e^{i \varphi / 2} & 0
\end{array}\right) \\
\hat{I}_{r} & =\frac{J_{r}}{2 i}\left(\begin{array}{cc}
0 & e^{i \varphi / 2} \\
-e^{-i \varphi / 2} & 0
\end{array}\right)
\end{aligned}
$$

The expectation value of the current is given by $I_{k} \equiv$ $\operatorname{Tr}\left(\hat{\rho} \hat{I}_{k}\right)=I_{d, k}+I_{p, k}$, where we have singled out a dynamic contribution $I_{d, k}=\rho_{g g}\left\langle g\left|\hat{I}_{k}\right| g\right\rangle$ and a geometric contribution $I_{p, k}=2 \Re \mathrm{e}\left(\rho_{g e}\left\langle e\left|\hat{I}_{k}\right| g\right\rangle\right)$. While $I_{d, k}$ relates to the usual supercurrent flowing in the presence of a phase bias, $I_{p, k}$ encapsulates the effects of the parametric drive (note that $\rho_{g e}=0$ for time-independent parameters) and is thus identified with the pumped current. The total charge transferred through the $k$-th SQUID in a period is given by $Q_{\mathrm{tr}, k}=\int_{0}^{T} I_{k}(t) d t$. Once again it is possible to distinguish a dynamic charge $Q_{d, k}=\int_{0}^{T} I_{d, k}(t) d t$ and a geometric (pumped) charge $Q_{p, k}=\int_{0}^{T} I_{p, k}(t) d t$, so that $Q_{\mathrm{tr}, \mathrm{k}}=Q_{d, k}+Q_{p, k}$. Adiabatic evolution and charge conservation force all three types of charges to be equal for the left and right SQUID. For this reason, we will safely drop the subscript $k$ in the following.

As first shown in Ref. 3, $Q_{d}$ and $Q_{p}$ are related to the derivative with respect to $\varphi$ of the dynamic phase $\Theta_{\mathrm{d}}$ and the geometric (Berry) phase $\Theta_{B}$ accumulated by the instantaneous ground state along a pumping cycle:

$$
\begin{aligned}
Q_{d} & =\frac{\partial \Theta_{d}}{\partial \varphi}, \\
Q_{p} & =\frac{\partial \Theta_{B}}{\partial \varphi} .
\end{aligned}
$$

Experimentally, $Q_{d}$ and $Q_{p}$ can be distinguished as they obey different symmetries. In particular upon reversing the direction of the pumping, $Q_{d}$ is not affected, while $Q_{p}$ duly changes its sign. We shall henceforth assume that such a distinction can be made and only be concerned with $Q_{p}$.

\section{FLUX PUMPING}

The principle of FP is illustrated in Fig. 1 (b), showing the time evolution of the control parameters during
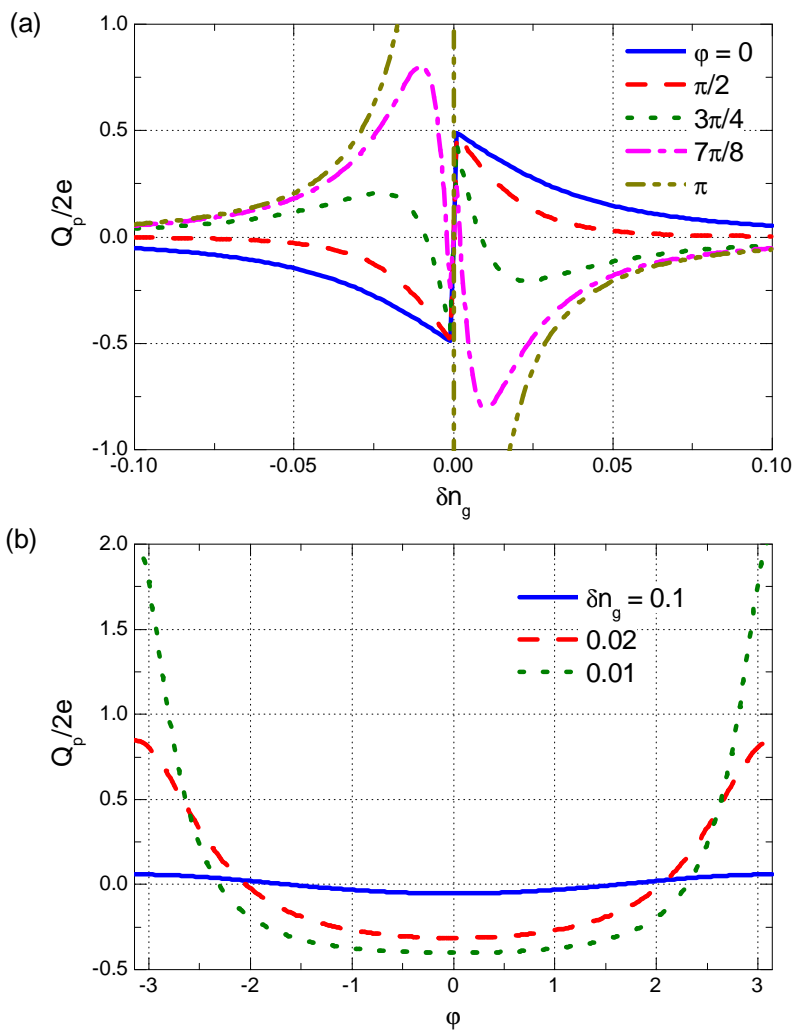

FIG. 2. (Color online) Pumped charge in the adiabatic limit. (a) Pumped charge $Q_{p}$ versus offset charge $\delta n_{g}$ for different values of the superconducting phase bias $\varphi$. (b) $Q_{p}$ versus $\varphi$ for different values of $\delta n_{g}$.

a pumping cycle. The charge offset $\delta n_{g}$, not shown, is set close to the degeneracy point (that is, $\left|\delta n_{g}\right| \ll 1$ ) and kept fixed throughout the cycle. At the initial time $t=0$, the island is decoupled from both leads, with $J_{l}$ and $J_{r}$ set to their minimum value $J_{\min }$. In sector I $(0 \leq t<T / 3)$, the coupling to the left lead is turned on by maximizing $J_{l}$. In sector II $(T / 3 \leq t<2 T / 3)$, the coupling is swapped from the left to the right lead, in such a way that the sum $J_{l}+J_{r}$ is kept constant. Finally, in sector III $(2 T / 3 \leq t<T) J_{r}$ is turned down to $J_{\text {min }}$, bringing the system back to the initial state. We have chosen linear ramps and a perfect coupling swap only for simplicity in deriving analytical expressions. As it goes with geometric pumping, a moderate tweaking of the pulses will not disrupt the pumping process as long as the solid angle spanned in the parameter space [see App. B stays approximately the same.

In the following, we will use the formalism of Sec. II to understand the adiabatic dynamics generated by FP and the corresponding pumped charge. We first discuss the case $\varphi=0$, as it allows for an intuitive explanation. For simplicity, we also set $J_{\min }=0$. 


\section{A. The case $\varphi=0$}

At $t=0$, the island is in a definite charge state $(0$ if $\delta n_{g}<0,1$ if $\delta n_{g}>0$ ) and the energy difference between charge states is $2 E_{C} \delta n_{g}$. As $J_{l}$ increases so that $J_{l} \gtrsim E_{C} \delta n_{g}$, the ground state evolves into a superposition of charge states. As a result, charge flows from the left lead onto the island. The charge transferred in this case is simply given by $\left|\left\langle 1 \mid g\left(\frac{T}{3}\right)\right\rangle\right|^{2}-|\langle 1 \mid g(0)\rangle|^{2}$. In sector II, the swapping of the couplings does not change the Hamiltonian (11). As a result, $\rho_{g e}=0$ and no charge is transferred. Finally, in sector III, the same amount of charge is released to the right lead as the system comes back (up to a geometric phase) to the initial state. Some plots of the instantaneous geometric currents $I_{p, l}, I_{p, r}$ are shown in App. A.

In this scheme, the fact that the dynamics is coherent plays a crucial role. This marks a clear difference between FP and previous pumping protocols. In the latter Cooper-pair tunneling is made energetically favorable via modulation of electrostatic potentials, so that the coherent-versus-incoherent nature of the tunneling process has only a modest influence on the pumped charge. By contrast, in FP there are no "pistons" pulling the Cooper-pairs around. As a result, one can show that in the limit of incoherent Cooper-pair tunneling the pumped charge vanishes.

The total pumped charge for the case $\varphi=0$ can be inferred from this heuristic argument, calculated by direct integration of $I_{p, k}$ [see Sec. II], or obtained by virtue of (9) [see App. B]. The result is:

$$
Q_{p}[\varphi=0]=-\frac{1}{2} \operatorname{sgn}\left(\delta n_{g}\right)\left(1-\frac{1}{\sqrt{1+r^{2}}}\right),
$$

where we have introduced the ratio

$$
r=\frac{J_{\max }}{2 E_{C} \delta n_{g}} .
$$

In the limit $r \rightarrow \pm \infty$ (corresponding to $E_{C}\left|\delta n_{g}\right| \ll$ $J_{\max }$ ), the absolute value of $Q_{p}$ approaches a maximum of half a Cooper pair. This result is approximately valid also for a finite $J_{\min }$, as long as $J_{\min } \ll E_{C} \delta n_{g}$.

The dependence of $Q_{p}$ on $\delta n_{g}$ exhibits a sawtooth behavior, as shown by the solid line in Fig. 2(a). At $\delta n_{g}=0$, the pumped charge changes sign discontinuously. However, when $\delta n_{g} \rightarrow 0$ also the minimum energy gap $\Delta E_{\min } \equiv \min _{t \in[0, T]} \Delta E(t)=\delta n_{g} E_{C}$ tends to zero. This implies that the adiabatic limit, in which the present derivation is valid, is only attained for infinitely slow evolution. We will return to this point in Sec. IV

\section{B. The general case}

When $\varphi \neq 0$, the same calculation leading to 10 shows that the pumped charge in sectors I and III is the same as in the case $\varphi=0$. This is only to be expected: as long as the island is only coupled to a single lead, the phase difference between the leads cannot play any role. The situation is different for sector II, where the coupling swap now takes place between two leads at different phases. As a result, an adjustment of the superconducting order parameter on the island is required 37 This causes an additional geometric current to flow across the sluice, in a direction opposite to that of the pumping.

In Fig. 2(a) we plot $Q_{p}$ versus $\delta n_{g}$ for different values of $\varphi$. For values of $\varphi$ in the range of 0 and $\pi / 2, Q_{p}$ simply decreases with respect to the case $\varphi=0$. As $\varphi$ is further increased, however, a new trend emerges: $Q_{p}$ changes its sign with respect to the unbiased case, except in the vicinity of the degeneracy point. The magnitude of the counterflowing $Q_{p}$ can well exceed a Cooper pair. Finally, at $\varphi=\pi$ the sign of the pumped charge is opposite to that of the unbiased case for all values of $\delta n_{g}$. Furthermore, $Q_{p}$ diverges as $1 / \delta n_{g}$ for $\delta n_{g} \rightarrow 0$.

The full dependence of $Q_{p}$ on $\varphi$ is shown in Fig. 2(b) for three selected values of $\delta n_{g}$. The reader may guess that the integral of each curve in Fig. 2(a) vanishes. Indeed, using (9) we obtain $\left\langle Q_{p}\right\rangle_{\varphi} \equiv \frac{1}{2 \pi} \int_{0}^{2 \pi} Q_{p}(\varphi) d \varphi=$ $\Theta_{B}(2 \pi)-\Theta_{B}(0)$. Even if in general $\Theta_{B}$ does not have to be single-valued, ${ }^{[7}$ in the present case $\Theta_{B}(2 \pi)=\Theta_{B}(0)$, so that $\left\langle Q_{p}\right\rangle_{\varphi}=0$. This implies that FP can only be observed in the presence of a well-defined phase bias, for if $\varphi$ randomly fluctuates in time, then no net charge is transferred on average. This is a clear signature of the coherent nature of the pumping process. On the other hand, $Q_{p}$ exhibits some degree of robustness against small phase fluctuations. In particular, for $\delta n_{g} \ll 1$, $Q_{p}$ develops a plateau centered at $\varphi=0$. This can be seen in the increased flattening of the curves with smaller $\delta n_{g}$ in Fig. 2 b) (an analytical argument is provided in App. B.

We remark that these features are peculiar to FP. To draw a comparison, let us recall that in "ordinary" Cooper-pair pumping $\left\langle Q_{p}\right\rangle_{\varphi}=1$, the phase dependence of $Q_{P}$ only appears as first-order correction in the small parameter $J_{\min } / J_{\max }$, and no significant dependence on the charge offset $\delta n_{g}$ is found as long as the gate modulation crosses the degeneracy point.

\section{The case $\varphi=\pi$}

We now fix our attention on the case $\varphi=\pi$, for which we can present analytical results. Upon direct integration of the current operator, we obtain for the charge pumped in the sector II

$$
Q_{p}^{(\mathrm{II})}[\varphi=\pi]=\operatorname{sgn}\left(\delta n_{g}\right) \frac{r^{2}}{2 \sqrt{1+r^{2}}} .
$$

This must be added to the contribution 100 from sectors I,III, to give the total pumped charge

$$
Q_{p}[\varphi=\pi]=\frac{1}{2} \operatorname{sgn}\left(\delta n_{g}\right)\left(\sqrt{1+r^{2}}-1\right) .
$$


An alternative derivation of 13 involving the Berry phase is shown in App. B. On comparing (10) and (13), we see that the pumping direction for $\varphi=\pi$ is always opposite to that for $\varphi=0$. From (13), it is apparent that $Q_{p}$ diverges for $\varphi=\pi$ and $r \rightarrow \infty$ (or $\delta n_{g} \rightarrow 0$ ). Notably, one finds that this divergence is not removed even when relaxing the constraint $J_{\min }=0$.

As mentioned, the present results have been derived in the adiabatic limit. So far, we have not investigated how tight a requirement this imposes on the driving frequency. To do so, we first notice that the adiabatic condition $\alpha \ll 1$ is equivalent to $\rho_{g e} \ll 1$. Now for $\varphi=\pi,\left|\rho_{g e}\right|$ is maximum at $t=T / 2$, where it attains the value

$$
\max _{0 \leq t<T}\left|\rho_{g e}\right|=\frac{3 r^{2}}{J_{\max } T} .
$$

Eq. (14) implies that when approaching the degeneracy point, the pumping period should be increased according to $T \propto r^{2}$ in order to stay in the adiabatic limit. In other words, the increase in $Q_{p}$ comes at the cost of an increasingly long $T$. We discuss this point in more detail in App. C. One can also check that the pumping current $I_{p}$ does not diverge at any time. In fact, $I_{p}$ never exceeds $I_{\max } \approx \frac{J_{\max }}{4} \rho_{g e} \ll J_{\max }$, that is, much less than the critical current of the SQUIDs.

\section{ADIABATIC BREAKDOWN AND DECOHERENCE}

In Sec. III, we carried out our calculations assuming adiabatic evolution during the pumping cycle. In this limit, the pumped charge is uniquely determined by the loop described in the parameter space of $\hat{H}$. As such, it does not depend on the pumping frequency. We also pointed out, however, that the validity of the adiabatic theorem requires the condition $\left|\rho_{g e}\right| \ll 1$ to hold. We then warned the reader that in the limit $\delta n_{g} \rightarrow 0$, due to the vanishing of the instantaneous energy gap at $t=0$ (and at $t=T / 2$ when $\varphi=\pi$ ), this condition requires the pumping cycle to be infinitely slow. As real measurements are always performed at finite frequencies, this implies that none of the traces of Fig. 2(a) can be exactly reproduced in an experiment in the vicinity of $\delta n_{g}=0$.

In this Section, we investigate the behavior of the pumped charge beyond the adiabatic limit. As soon as we allow nonadiabatic transitions to take place, the dynamics of the system becomes highly nontrivial. As argued elsewhere $e^{13|15| 26}$, the pumped charge is a sensitive probe of this dynamics. Indeed, it was exploited in Ref. 22 to characterize Landau-Zener transitions in the Cooper-pair sluice. Furthermore, the final state of the pump at the end of the pumping cycle is in general different from its initial state. As measurements are typically averaged over very many cycles, the quantity of experimental interest becomes the stationary pumped charge $Q_{p}^{\text {st }}$. The latter is determined by an interplay between the nonadiabatic drive and decoherence effects due
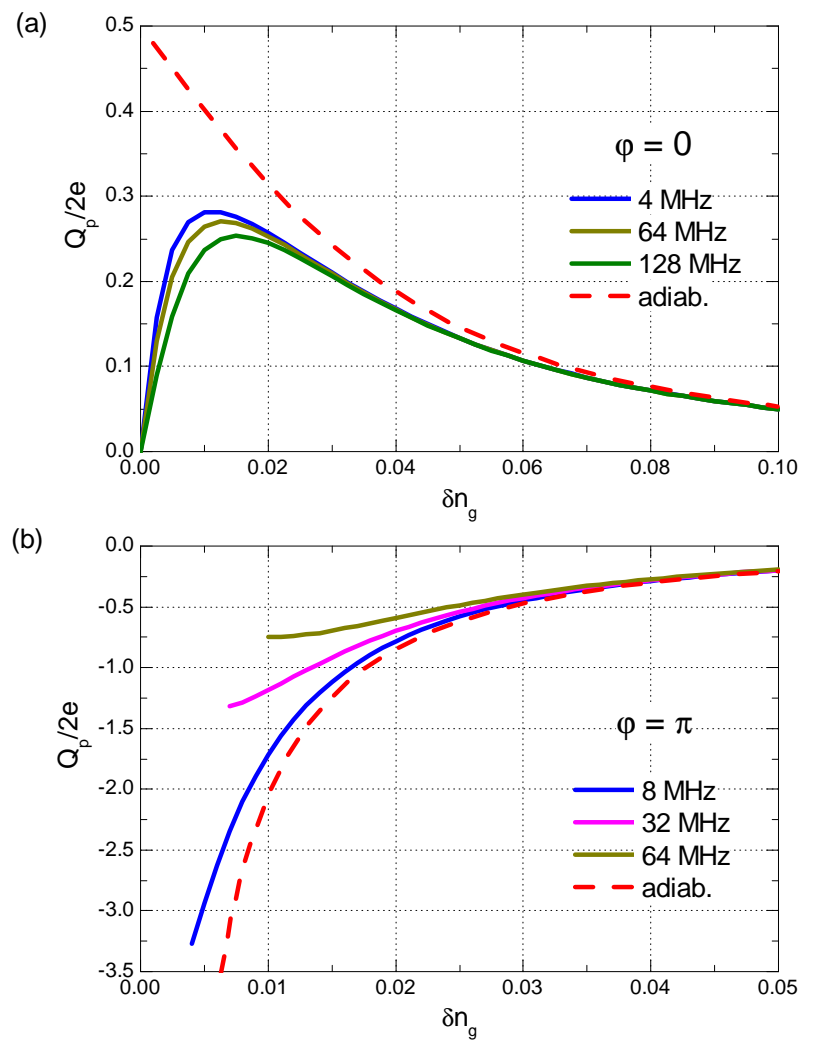

FIG. 3. (Color online) Breakdown of adiabatic behavior. $Q_{p}$ versus $\delta n_{g}$ for $\varphi=0$ (a) and $\varphi=\pi$ (b), for different pumping frequencies $f$ (solid lines). The results are obtained by numerical integration of the master equation of Ref. 26] The adiabatic-limit predictions $(10)$ and $\sqrt{13}$ are also plotted for comparison (dashed lines). Smooth pulses are used in place of those of Fig. 1(b) for improved adiabaticity. Relevant parameter values for the pump are: $E_{C}=1 \mathrm{~K}, J_{\max }=0.1 E_{C}, J_{\min }=$ $0.03 J_{\max }$. For the fictitious environment (see Ref. 27 for details): $g=0.02, R=300 \mathrm{k} \Omega, T=0, T_{0}=0.4 \mathrm{~K}$.

to the electromagnetic environment in which the pump is embedded. A full characterization of such effects is beyond the scope of this work. Still, the inclusion of decoherence in the model is essential in order to reach a quasistationary state.

We present numerical results obtained using the master equation approach developed in Refs. 26 and 27, which consistently accounts for the combined action of a quasiadiabatic drive and decoherence on an open quantum two-level system. This formalism is not intended to address the fully nonadiabatic case; yet, it can be conveniently used to investigate the parameter region where the adiabatic condition ceases to hold. Decoherence (dephasing and relaxation) is modeled by attaching a fictitious environment to the pump, in the form of a resistor capacitively coupled to the central island. This mimics the effect of charge noise, which is known to be the first cause of decoherence in charge-based devices ${ }^{38}$.

In Refs. 26 and 27 it was found that a zero-temperature 
environment tends to stablize ground-state pumping, effectively extending the region of adiabaticity. Here we also consider a zero-temperature enviroment. By tuning the coupling parameter to a small value, we make sure that nonadiabatic transitions still play the major role. In this case, decoherence only acts as a weak source of dissipation: it damps the oscillations in the pumped charge and slowly brings the system into a quasistationary state.

In Fig. 3 we plot $Q_{p}^{\text {st }}$ versus $\delta n_{g}$ for the emblematic cases $\varphi=0$ (a) and $\varphi=\pi$ (b). We choose the realistic device parameters $E_{C}=1 \mathrm{~K}, J_{\max }=0.1 E_{C}, J_{\min }=$ $0.03 J_{\max }$, use smooth pulses instead of those in Fig. 1 ( (b) and explore different pumping frequencies (solid lines). The adiabatic-limit predictions for the two cases (Eqs. 10 and 13. respectively) are also plotted for comparison (dashed lines); notice, however, that they were derived in the limit $J_{\min } \rightarrow 0$.

In general, nonadiabatic transitions result in a decrease of $Q_{p}$. This is qualitatively accounted for by the fact that the adiabatic excited state of the sluice carries an opposite $Q_{p}$ with respect to the ground state. For the case $\varphi=0$ [Fig. 3(a)], this results in a smearing of the adiabatic sawtooth. Note that since we have considered the realistic case $J_{\min } \neq 0$, in the limit $\delta n_{g} \rightarrow 0$ one still has $\Delta E_{\min }=J_{\min }$, so that the residual coupling partly holds back nonadiabatic transitions. Analogous considerations can be made for the case $\varphi=\pi$ [Fig. 3(b)]. As $\delta n_{g}$ is reduced, however, the nonadiabatic behavior is no longer mitigated by the presence of a finite $J_{\min }$ (this relates to the fact that $E_{12}$ vanishes for $J_{l}=J_{r}$ when $\varphi=$ $\pi$, see Eq. 3a). The effect is thus more dramatic, with higher frequencies hitting the nonadibatic onset first. We terminate each data series as soon as $\rho_{g e}$ exceeds the arbitrary threshold 0.3 ; further points would fall outside the range of validity of our master equation.

These results indicate that nonadiabatic effects must be taken into serious consideration in any pratical implementation of FP. A relevant figure of merit for optimization is the average geometric current $\left\langle I_{p}\right\rangle=f Q_{p}$ $(f=1 / T)$, as this is the signal to be detected in a realistic readout scheme. An example of such optimization is presented in App. C for the case $\varphi=\pi$.

\section{CONCLUSIONS}

We have presented a new scheme for Cooper-pair pumping, flux pumping (FP). Based on magnetic-flux control, FP uses neither a bias voltage nor a modulation of gate voltages. FP is realized by coherent transer of a superposition of charge states across a superconducting island. The resulting pumped charge depends on the gate position and on the phase difference across the pump in a distinctive fashion. As no incoherent process can mimic these features, their witnessing would be an unambiguous demonstration of purely coherent Cooper-pair pumping.

The implementation of FP looks feasible, expecially in the light of recent results obtained with the Cooper-pair sluice ${ }^{20 \mid 22}$. An apparent matter of concern is the fact that the supercurrent flowing through the device may well exceed the pumped current. For instance, let us take the device parameters of Fig. 3 and the pumping cycle of Fig. 11(a). The mean dynamic current at $\varphi=\pi / 2$ can be approximated by $\left\langle I_{d}\right\rangle \approx \frac{2 e}{24 \hbar} J_{\max } \approx 350 \mathrm{pA}$, independent of frequency. Now at a typical $f=120 \mathrm{MHz}, Q_{p}=e$ corresponds to $\left\langle I_{p}\right\rangle \approx 20 \mathrm{pA}$, so that the pumped current accounts for less than $10 \%$ of the signal. This is not an issue, however, as the supercurrent term is even with respect to time-reversal symmetry, while the pumped current is odd. As a result, $\left\langle I_{p}\right\rangle$ can be determined by simply subtracting the measured currents when pumping in opposite directions (as done in Ref. 20).

Still, detecting such a small current circulating in a loop may challenge customary techniques. In the search for signatures of FP, an important role is likely to be played by its distinctive symmetries. Besides timereversal, $\left\langle I_{p}\right\rangle$ is also an odd function of the gate position with respect to degeneracy. Finally, it should not depend on the direction of the circulating currents in the SQUIDs. Altogether, these symmetries may be used to rule out the contribution of undesired rectification effects, possibly originating from spurious inductive or capacitive couplings.

\section{ACKNOWLEDGMENTS}

The authors are grateful to T. Aref, L. Arrachea, F. Giazotto, J. Pekola, A. Shnirman, and P. Solinas for valuable discussions. This work was supported by the European Community FP7 under grant No. 238345 "GEOMDISS". S. G. acknowledges financial support from the Finnish National Graduate School in Nanoscience.

\section{Appendix A: Instantaneous geometric currents}

In Fig. 4 we plot the instantaneous geometric currents $I_{p, l}$ and $I_{p, r}$ for the cases $\varphi=0(\mathrm{a}), \pi / 2(\mathrm{~b})$, and $\pi$ (c). The current profiles in the first and third sector are the same for all three plots [notice the change of scale in panel (c)]. By contrast, the currents in sector II strongly depend on $\varphi$. A counterflowing current, absent for $\varphi=0$ [panel (a)] develops for finite $\varphi$ [panel (b)] and largely exceeds the forward current in magnitude as $\varphi$ approaches $\pi$ [panel (c)]. The discontinuities at sector boundaries are due to the cusps in the pulses of Fig. 1 (b) and disappear as soon as the latter are replaced by smooth pulses.

\section{Appendix B: Flux pumping and Berry phase}

The charge pumped by a superconducting pump in the adiabatic limit is linked to the Berry phase $\Theta_{B}$ accumulated by the instantaneous ground state along the pumping cycle $^{3}$, as prescribed by (9). For a two-level system 

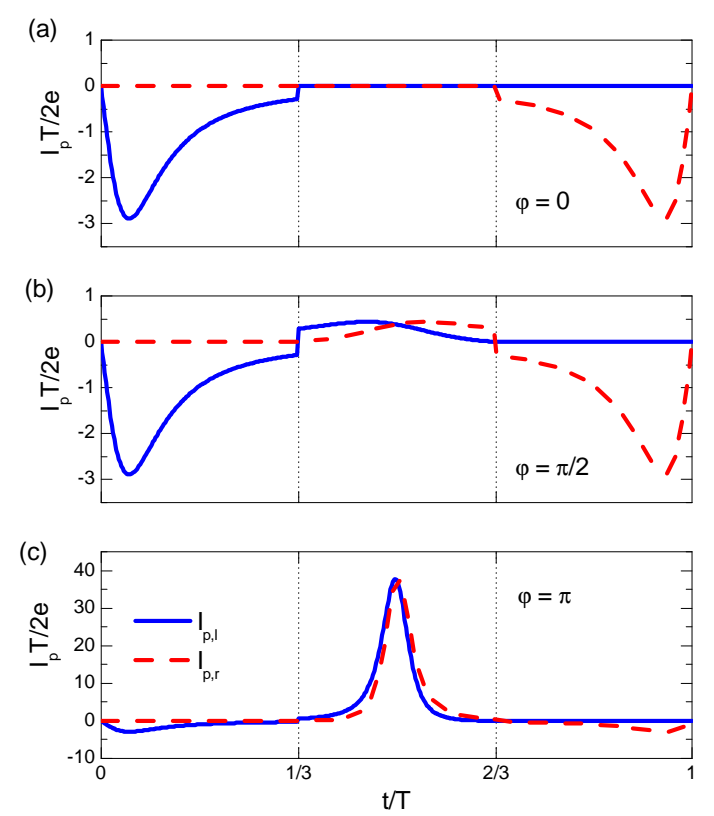

FIG. 4. (Color online) Instantaneous geometric currents. $I_{p, l}$ (solid line) and $I_{p, r}$ (dashed line) versus time for $\delta n_{g}=-0.02$ and $\varphi=0(\mathrm{a}), \pi / 2(\mathrm{~b})$, and $\pi(\mathrm{c})$.

parametrically driven in closed loop, $\Theta_{B}$ is proportional to the solid angle spanned by the Bloch vector, which performs an adiabatic rotation on the Bloch sphere. The path drawn by the Bloch vector is shown in Fig. 5 (a) for the pumping cycle of Fig. 1 (b) and a few selected values of $\varphi$. The resulting $\Theta_{B}$ is plotted versus $\varphi$ and $\delta n_{g}$ in Fig. 5(b). According to (9), the pumped charge $Q_{p}$ is given by the local slope of the surface plot along the $\varphi$ axis.

Using (3), (4), and the definition $\frac{23}{2}$, we find

$$
\Theta_{B} \equiv i \int_{0}^{T} d t\langle g \mid \dot{g}\rangle=\frac{1}{2} \int_{0}^{T} d t(1+\eta) \dot{\gamma}
$$

For the given pumping cycle, $\gamma(t)=-\varphi / 2$ in sector I and $\gamma(t)=\varphi / 2$ in sector III. The time derivative of $\gamma$ vanishes in these regions. The sudden change of $\gamma$ from $\varphi / 2$ to $-\varphi / 2$ at times $0, T, \ldots$ yields a delta function in the time derivative, but at that time $1+\eta=0$, so that the integrand in (B1) vanishes as well. As a result, the only contribution to $\Theta_{B}$ comes from sector II. The fact that sectors I and III do not contribute to $\Theta_{B}$ is a consequence of our choice of adiabatic basis (Eqs. 4), and is not in contrast with the fact that there is a charge flow in sectors I, III. Indeed, $\Theta_{B}$ is only defined for closed loops. It is possible to give a gauge-invariant generalization of the Berry phase for open loops ${ }^{39}$, but we do not need it here.

We will now explicitly calculate $\Theta_{B}$ and $Q_{p}$ in two (a)
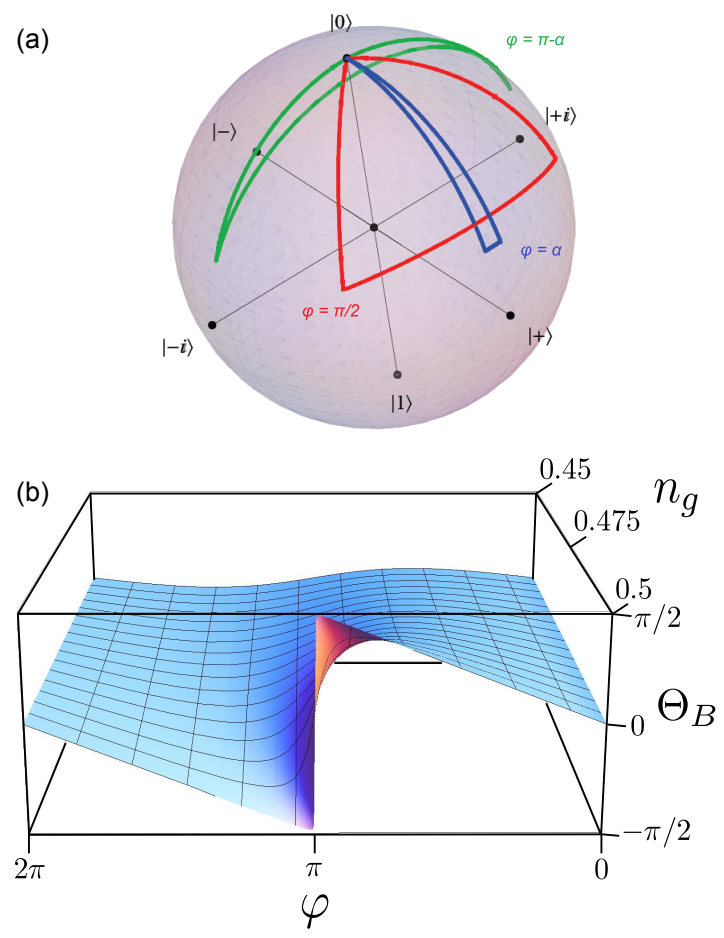

FIG. 5. (Color online) Pumping cycle and Berry phase. (a) Plots of the path drawn by the ground state of the sluice on the Bloch sphere along a pumping cycle, for $\varphi=\alpha$ (blue), $\varphi=\pi / 2$ (red), and $\varphi=\pi-\alpha$ (green), with $\alpha \ll 1$. The Berry phase is proportional to the solid angle spanned by the paths. (b) Berry phase $\Theta_{B}$ versus $\varphi$ and $\delta n_{g}$. According to (9), The pumped charge $Q_{p}$ is proportional to the slope of the surface plot along the $\varphi$ axis.

important cases.

\section{Case $\varphi=0$}

For definiteness, we assume $\delta n_{g}<0$. To calculate $Q_{p}$ for $\varphi=0$, it is sufficient to expand $\Theta_{B}$ to first order in $\varphi$. Up to this order, $\eta$ is time-independent in sector II, so that $\Theta_{B} \approx \frac{1}{2}(1+\eta)[\gamma(2 T / 3)-\gamma(T / 3)]=\frac{1}{2}(1+\eta) \varphi$. The pumped charge is thus

$$
Q_{p}[\varphi=0]=\frac{1}{2}(1-\eta)
$$

as we found in 10 .

It is worth noting that near the degeneracy point $\delta n_{g} \ll 1$, the validity of $\mathrm{B} 2$ ) extends to all phases $\varphi \neq \pi$. In fact, for sufficiently small $\delta n_{g}, \eta \approx 0$ for all times in sector II. As a result, the pumped charge is half a Cooper pair, as predicted by $(\mathrm{B} 2)$. 


\section{Case $\varphi=\pi$}

Using (9) and (B1), we find for the pumped charge

$$
\begin{aligned}
Q_{p}[\varphi=\pi]= & \frac{1}{2} \int_{T / 3}^{2 T / 3} d t\left[1+\frac{\delta n_{g}}{\sqrt{\delta n_{g}^{2}+\left[\left(J_{l}-J_{r}\right) /\left(2 E_{C}\right)\right]^{2}}}\right] \\
& \times \frac{\partial^{2}}{\partial t \partial \varphi} \arctan \left(\frac{J_{r}-J_{l}}{J_{r}+J_{l}} \tan \frac{\varphi}{2}\right) .
\end{aligned}
$$

Using $J_{r}+J_{l}=J_{\max }$ and $J_{r}-J_{l}=6 J_{\max } u / T$ with $u=t-T / 2$, the integral can be evaluated analytically to give

$$
\begin{aligned}
Q_{p}[\varphi=\pi] & =\frac{T}{12} \int_{0}^{T / 6} \frac{d u}{u^{2}}\left[1-\frac{1}{\sqrt{1+(6 r u / T)^{2}}}\right] \\
& =\frac{1}{2}\left[\sqrt{1+r^{2}}-1\right] .
\end{aligned}
$$

\section{Appendix C: Adiabatic breakdown and optimal pumping frequency}

Here we present a numeric optimization of the average pumped current $\left\langle I_{p}\right\rangle$ for the case $\varphi=\pi$.

In Fig. 6(a) we plot $\left\langle I_{p}\right\rangle$ versus $f$ for different values of $\delta n_{g}$ in the range of 0.005 and 0.1 (from left to right). The fact that each curve reaches a maximum indicates that there is a tradeoff between speed-up gain and adiabaticity loss. For each value of $\delta n_{g}$, the optimal frequency $f^{*}\left(\delta n_{g}\right)$ and the corresponding maximum current $I^{*}\left(\delta n_{g}\right)$ are plotted in Figs. 66(b) and 6(c), respectively. From Fig. 6(b) we see that the optimal frequency is approximately proportional to $\delta n_{g}$. From Fig. 6(c), that $I^{*}$ attains its maximum at a finite $\delta n_{g}$. In particular, this shows that the optimal operation point is not arbitrarily close to $\delta n_{g}=0$, as one might erroneously guess before taking nonadiabatic corrections into account. The scaling of both $f^{*}$ and $I^{*}$ is approximately linear as $\delta n_{g} \rightarrow 0$, as demonstrated by the linear fits shown as dashed lines in panels $(b, c)$.
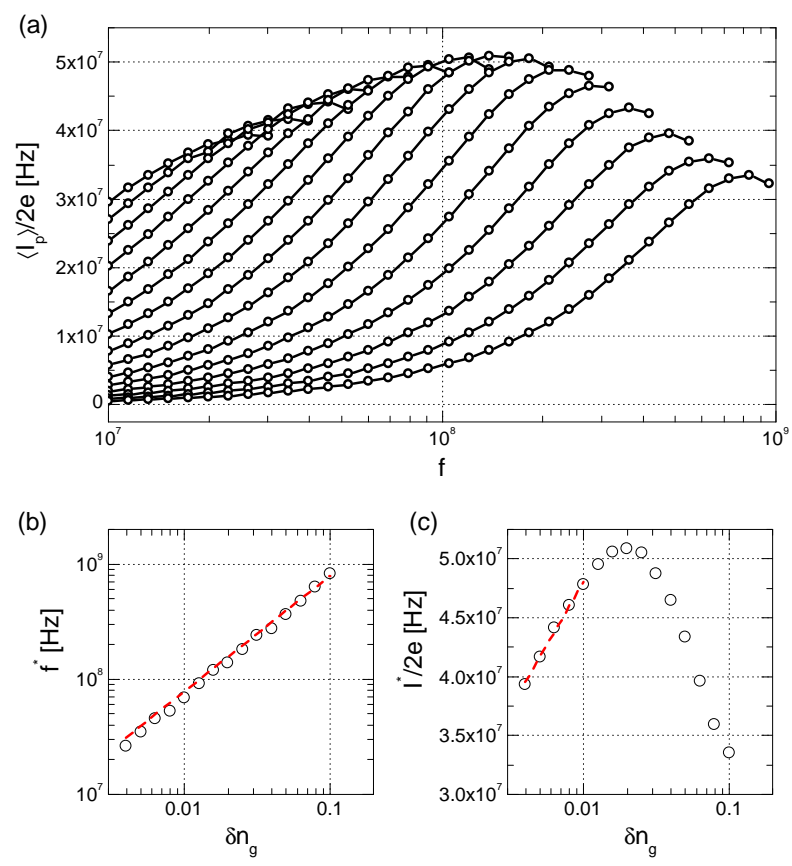

FIG. 6. Optimal pumping frequency when $\varphi=\pi$. (a) Average pumped current $\left\langle I_{p}\right\rangle$ versus pumping frequency $f$ with $\varphi=\pi$ and $\delta n_{g}$ taking a set of values in the range of 0.005 and 0.1 (from left to right). The other parameters are the same as in Fig. 3 (b,c) Optimal pumping frequency $f^{*}(\mathrm{~b})$ and corresponding maximum pumped current $I^{*}$ (c) versus $\delta n_{g}$. The dashed lines in (b) and (c) are linear fits to the numeric data close to $\delta n_{g}=0$. 
1 J. P. Pekola, J. J. Toppari, M. Aunola, M. T. Savolainen, and D. V. Averin, Phys. Rev. B 60, R9931 (1999).

${ }^{2}$ L. Y. Gorelik, A. Isacsson, Y. M. Galperin, R. I. Shekhter, and M. Jonson, Nature 411, 454 (2001).

3 M. Aunola and J. J. Toppari, Phys. Rev. B 68, 020502(R) (2003).

4 A. O. Niskanen, J. P. Pekola, and H. Seppä, Phys. Rev. Lett. 91, 177003 (2003).

${ }^{5}$ R. Fazio, F. W. J. Hekking, and J. P. Pekola, Phys. Rev. B 68, 054510 (2003).

6 A. Romito, F. Plastina, and R. Fazio, Phys. Rev. B 68, 140502 (2003)

7 M. Möttönen, J. P. Pekola, J. J. Vartiainen, V. Brosco, and F. W. J. Hekking, Phys. Rev. B 73, 214523 (2006).

8 M. Cholascinski and R. W. Chhajlany, Phys. Rev. Lett. 98, 127001 (2007).

9 S. Safaei, S. Montangero, F. Taddei, and R. Fazio, Phys. Rev. B 77, 144522 (2008).

10 R. Leone, L. P. Lévy, and P. Lafarge, Phys. Rev. Lett. 100, 117001 (2008).

11 V. Brosco, R. Fazio, F. W. J. Hekking, and A. Joye, Phys. Rev. Lett. 100, 027002 (2008).

12 J.-M. Pirkkalainen, P. Solinas, J. P. Pekola, and M. Möttönen, Phys. Rev. B 81, 174506 (2010).

13 S. Gasparinetti, P. Solinas, and J. P. Pekola, Phys. Rev. Lett. 107, 207002 (2011).

14 I. Kamleitner and A. Shnirman, Phys. Rev. B 84, 235140 (2011).

15 J. Salmilehto and M. Möttönen, Phys. Rev. B 84, 174507 (2011).

16 J. Salmilehto and M. Möttönen, Phys. Rev. B 86, 184512 (2012).

17 L. J. Geerligs, S. M. Verbrugh, J. E. Mooij, H. Pothier, C. Urbina, and M. H. Devoret, Z. Phys. B 85, 349 (1991).

18 A. O. Niskanen, J. M. Kivioja, H. Seppä, and J. P. Pekola, Phys. Rev. B 71, 012513 (2005).

19 J. J. Vartiainen, M. Möttönen, J. P. Pekola, and A. Kemppinen, Appl. Phys. Lett. 90, 082102 (2007).

20 M. Möttönen, J. J. Vartiainen, and J. P. Pekola, Phys. Rev. Lett. 100, 177201 (2008).

21 F. Hoehne, Y. A. Pashkin, O. V. Astafiev, M. Möttönen, J. P. Pekola, and J. S. Tsai, Phys. Rev. B 85, 140504
(2012)

22 S. Gasparinetti, P. Solinas, Y. Yoon, and J. P. Pekola, Phys. Rev. B 86, 060502(R) (2012).

${ }^{23}$ M. V. Berry, Proc. R. Soc. Lond. A 392, 45 (1984).

24 B. Simon, Phys. Rev. Lett. 51, 2167 (1983).

25 S. N. Shevchenko, S. Ashhab, and F. Nori, Phys. Rep. 492, 1 (2010).

26 J. P. Pekola, V. Brosco, M. Möttönen, P. Solinas, and A. Shnirman, Phys. Rev. Lett. 105, 030401 (2010).

27 P. Solinas, M. Möttönen, J. Salmilehto, and J. P. Pekola, Phys. Rev. B 82, 134517 (2010).

28 A. Russomanno, S. Pugnetti, V. Brosco, and R. Fazio, Phys. Rev. B 83, 214508 (2011).

29 P. W. Brouwer, Phys. Rev. B 58, 10135 (1998).

30 M. Switkes, Science 283, 1905 (1999).

${ }^{31}$ F. Giazotto, P. Spathis, S. Roddaro, S. Biswas, F. Taddei, M. Governale, and L. Sorba, Nat. Phys. 7, 857 (2011).

${ }^{32}$ K. Eckert, M. Lewenstein, R. Corbalán, G. Birkl, W. Ertmer, and J. Mompart, Phys. Rev. A 70, 023606 (2004).

33 A. D. Greentree, J. H. Cole, A. R. Hamilton, and L. C. L. Hollenberg, Phys. Rev. B 70, 235317 (2004).

34 J. Siewert, T. Brandes, and G. Falci, Opt. Comm. 264, 435 (2006).

35 I. Kamleitner, J. Cresser, and J. Twamley, Phys. Rev. A 77, 032331 (2008).

36 D. Vion, A. Aassime, A. Cottet, P. Joyez, H. Pothier, C. Urbina, D. Esteve, and M. H. Devoret, Science 296, 886 (2002).

37 This can be seen by introducing the operator $e^{\hat{i} \theta}$, related to the charge number operator $N$ by the commutation rule $\left[\hat{N}, e^{\hat{i} \theta}\right]=e^{\hat{i} \theta}$. Its representation in the reduced charge basis is given by $e^{i \hat{\theta}}=|1\rangle\langle 0|$. Using (4), one can show that $\left\langle g\left|e^{\hat{i} \theta}\right| g\right\rangle=e^{i \gamma}$. We thus identify $\gamma$ with the expectation value of the phase of the island. As expected, $\gamma$ equals the phase of the left lead at the beginning of sector II, and that of the right lead at its end.

38 G. Ithier, E. Collin, P. Joyez, P. J. Meeson, D. Vion, D. Esteve, F. Chiarello, A. Shnirman, Y. Makhlin, J. Schriefl, and G. Schön, Phys. Rev. B 72, 134519 (2005).

39 J. Samuel and R. Bhandari, Phys. Rev. Lett. 60, 2339 (1988). 